\title{
Aspectos Biométricos de las Arterias Cerebral Anterior en el Segmento Proximal (A1) y Carótida Interna
}

\author{
Biometrical Aspects of the Anterior Cerebral Artery in its Proximal Segment (A1) \\ and Internal Carotid Artery
}

*Eduardo Mandiola; "Eduardo Alarcón; *J. Carlos Oñate; *Pablo Sanhueza; **Mariano del Sol \& **Enrique Olave

MANDiOla, E.; ALARCón, E.; OÑATE, J. C., SANHUEZA, P.; DEL SOL, M. \& OLAVE, E. Características biométricas de las arterias cerebral anterior en el segmento proximal (A1) y carótida interna. Int. J. Morphol., 25(4):915-918, 2007.

RESUMEN: La conformación del círculo arterial cerebral tiene relevancia en la clínica neuroquirúrgica por la relación compleja que presentan las arterias que lo originan y su gran variabilidad Debido a a esto, hemos decidido efectuar un análisis biométrico de las arterias cerebral anterior (A1) y de la arteria carótida interna (ACI) por posible asociación o correlación en estas estructuras. El trabajo se realizó en 36 cerebros disponibles en los laboratorios y cuyos datos bioantropológicos estaban registrados. El segmento A1 de la arteria cerebral anterior derecha tuvo una longitud y calibre promedio de $12,86 \pm 1.58 \mathrm{~mm}$ y $2,37 \pm 0,68 \mathrm{~mm}$, respectivamente. En el lado izquierdo la longitud y el calibre promedio de esta arteria fue $12,62 \pm 1.96 \mathrm{~mm}$ y de $2,42 \pm 0,75 \mathrm{~mm}$, respectivamente. El calibre del lado derecho de la ACI fue $3,84 \pm 0,68 \mathrm{~mm}$ y del lado izquierdo fue $3,96 \pm 0,87 \mathrm{~mm}$. Se observó variabilidad anatómica de los componentes que constituyen el círculo arterial cerebral cuando se considera lado, sexo e índice cefálico.

PALABRAS CLAVE: Arteria cerebral anterior; Círculo arterial del cerebro; Arteria carótida interna.

\section{INTRODUCCION}

Es importante observar la relación compleja de las arterias que forman el círculo arterial del cerebro (Willis), especialmente, sus variaciones anatómicas. Éstas pueden en algunas ocasiones provocar alteraciones funcionales, cuando su conformación no es la adecuada para suplir las estructuras cerebrales, centrales y corticales, ya sea por alteraciones en su desarrollo o por otros factores que podrían afectarlas, como es el caso de los procesos expansivos.

A menudo las arterias comunicantes forman anastomosis funcionalmente inadecuadas, que son las causantes de la alta incidencia de serios trastornos en la irrigación, después de una oclusión unilateral o compresión de la carótida interna, especialmente en las personas de edad avanzada (Carpenter, 1978).

En la literatura anatómica y clínica, se menciona que existen numerosas variaciones tanto en el calibre como en el trayecto de las arterias componentes del círculo arterial cerebral (Duroux et al., 1953; González, 1959; Barbosa et al., 1969; Lazorthes et al., 1979; Kamath, 1981; Orlandini et al., 1985; Milisavljevic et al., 1986; Overbeeke et al., 1991).

Hillen \& Hoogstraten (1990) demostraron que las variaciones del calibre de los vasos, la edad y sexo, más factores genéticos, influyen en la hemodinámica de estas estructuras vasculares. Por otra parte, Gulisano et al., (1982) señalaron la necesidad de conocer con detalles el comportamiento de estas arterias, en lo relativo a padrones morfológicos.

Generalmente, los clínicos suponen una perfección anatómica del círculo arterial cerebral en los pacientes que evalúan, lo que no siempre es correcto por su gran variabilidad, observable en las arteriografías (Macchi et al., 2002) y hallazgos en el campo operatorio (Kim et al., 2002).

\footnotetext{
* Área de Anatomía Humana. Departamento de Ciencias Biològicas. Universidad Andrés Bello, Santiago, Chile.

${ }^{* *}$ Facultad de Medicina, Universidad de La Frontera, Temuco, Chile.

Parcialmente financiado por Proyecto DI-UNAB-02-05/R. Universidad Andrés Bello, Santiago, Chile.
} 
En trabajos previos hemos expresado, que son escasas las investigaciones que asocian características morfológicas vasculares encefálicas con caracteres bioantropológicos (Godinov, 1929; Mandiola et al., 1995-1999).

En este trabajo analizamos las características biométricas de las arterias carótida interna (ACI) y cerebral anterior (ACA) en el segmento A1 como parte de la estructura del círculo cerebral.

\section{MATERIAL Y MÉTODO}

Se estudiaron 36 encéfalos coleccionados en el Laboratorio de Anatomía con registros bioantropológicos disponibles, entre ellos, el índice cefálico (IC). Se analizaron los vasos encefálicos que forman el círculo arterial cerebral, especialmente la ACA en su segmento A1 y la ACI, registrando fotográficamente a estas estructuras. Los datos obtenidos en relación con las variables lado, sexo e índice cefálico (IC) fueron sometidos a cálculo de valores medio, desviación estándar y correlación. Se aplicó también, el t-de Student.

\section{RESULTADOS}

Con respecto al calibre de la ACA (A1) y considerando los lados, se obtuvo en el lado derecho un promedio de 2,37 \pm $0,68 \mathrm{~mm}$ y en el izquierdo $2,42 \pm 0,75 \mathrm{~mm}$ no se apreciándose diferencias significativas al comparar ambos lados (Fig. 1).

Cuando se consideró el IC el calibre promedio de la ACa fue de 2,34 \pm 0,54 mm para los braquicéfalos y 2,43 \pm 079 mm, para los dólico-mesocéfalos, no observándose diferencias significativas al comparar ambos grupos (Fig. 1).

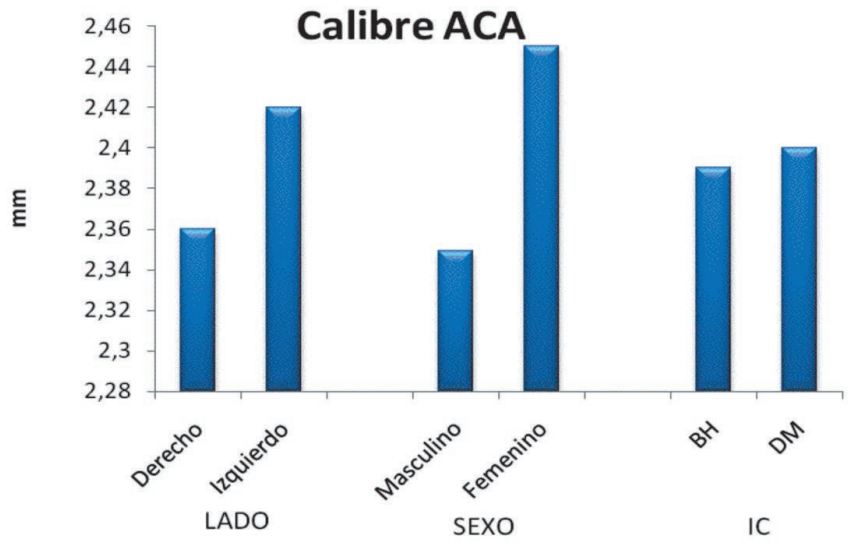

Fig. 1. Valores medios y desviación estándar del calibre de la arteria cerebral anterior en el segmento A1 en relación a lado, sexo e índice cefálico. (BH. Braquicéfalos; DM. Dólico-mesocéfalos)
Con respecto a la longitud promedio de la ACA en el lado derecho fue de $12,86 \pm 1,58 \mathrm{~mm}$ y en el lado izquierdo de 12,62 $\pm 1,96 \mathrm{~mm}$ (Fig. 2).

En relación al sexo, lo longitud promedio fue de 12,81 $\pm 1,86 \mathrm{~mm}$ en el masculino y de $12,57 \pm 1,58 \mathrm{~mm}$ en el femenino (Fig. 2).

Considerando el IC, el grupo BH presentó una longitud promedio de $13,03 \pm 2,11 \mathrm{~mm}$ y el DM de $12,58 \pm 1,57 \mathrm{~mm}$ (Fig. 2).

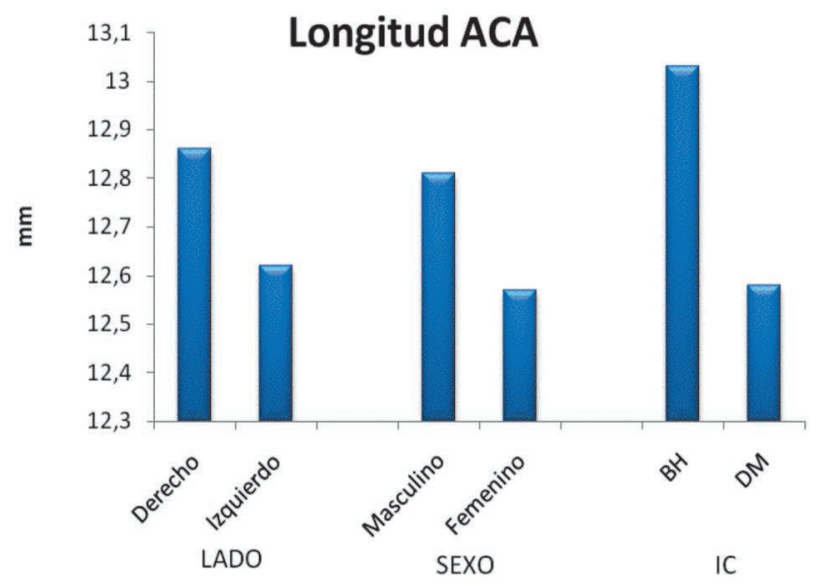

Fig. 2. Valores medios y desviación estándar de la longitud de la arteria cerebral anterior en el segmento A1 en relación a lado, sexo e índice cefálico.

El calibre promedio de la ACI fue de $3.84 \pm 0,68 \mathrm{~mm}$ en el lado derecho y de $3,96 \pm 0,87 \mathrm{~mm}$ en el lado izquierdo, no observándose diferencias estadísticamente significativas $(\mathrm{p}=0,2486)$ al comparar ambos lados (Fig. 3).

Al considerar la variable sexo, el calibre promedio de la ACI fue de 4,00 $\pm 0,91 \mathrm{~mm}$ en el femenino y de $3,86 \pm 0,73 \mathrm{~mm}$ en el masculino. No hubo diferencias estadísticamente significativas al comparar ambos grupos (Fig. 3).

En lo que respecta al IC, en los $\mathrm{BH}$ el calibre promedio de la ACI fue de 4,09 $\pm 0,65 \mathrm{~mm}$ y en los DM $3,80 \pm 0,83 \mathrm{~mm}$. Al comparar ambos grupos no hubo diferencias estadísticamente significativas $(\mathrm{p}=0,1384)$.

Las comparaciones estadísticas de los parámetros lado, sexo e IC arrojó diferencias no significativas.

Una observación importante corresponde al análisis de correlación entre el calibre de la ACI y la ACA donde se observó una correlación positiva $(\mathrm{p}=0.0003)$. (Fig. 4). 


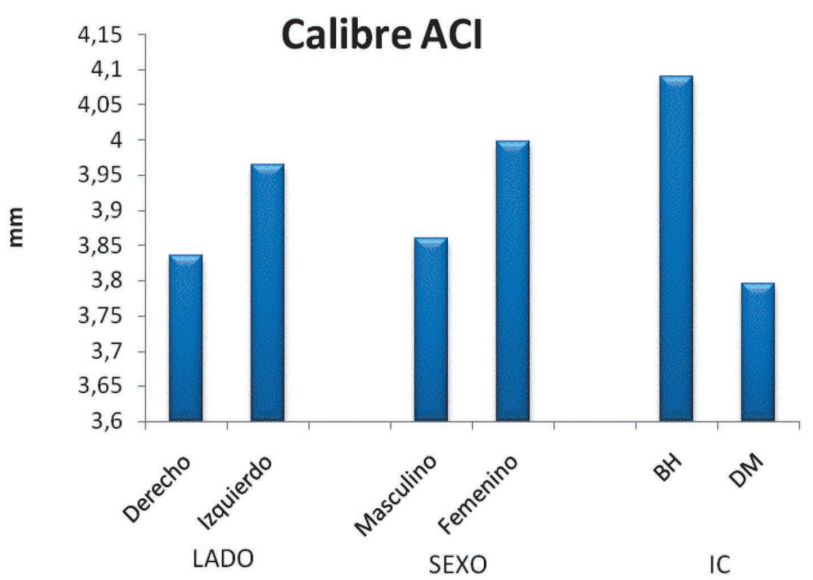

Fig. 3. Valores medios y desviación estándar del calibre de la arteria carótida, en relación a lado, sexo e índice cefálico.

Correlación calibre A. Carotida v/s ACA (A1)

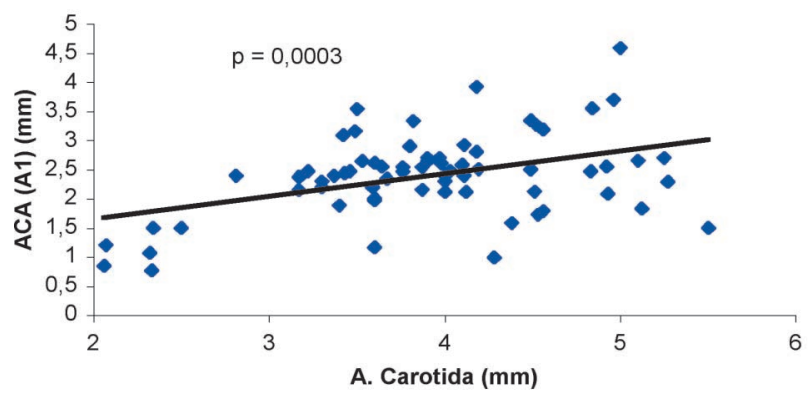

Fig. 4. Curva de correlación de los calibres entre las arterias carótida interna y la arteria cerebral anterior en el segmento A1.

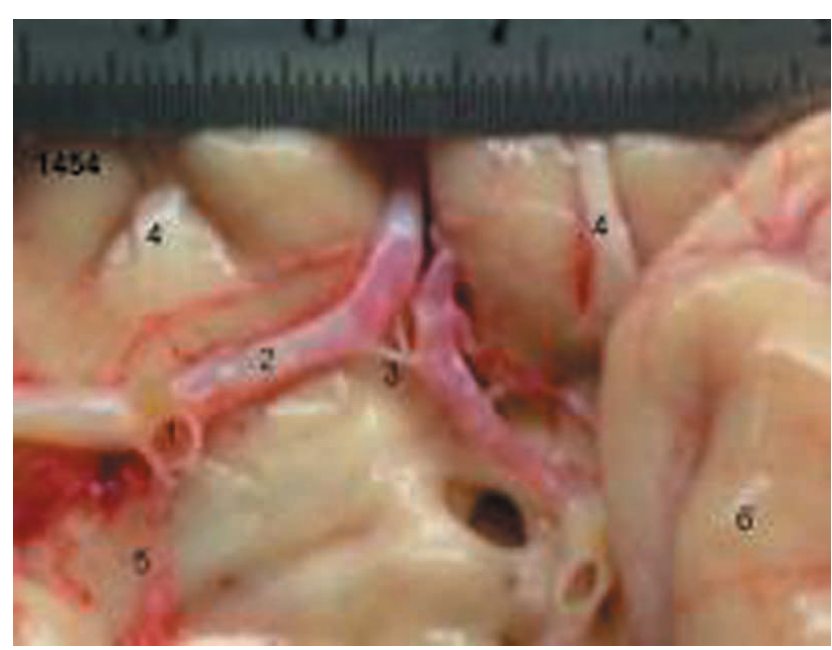

Fig. 5. Visión ventral de la parte superior del círculo arterial cerebral. 1. A. Carótida interna. 2. A. Cerebral anterior. 3. A. Comunicante anterior doble e hipoplástica. 4. Tracto olfatorio. 5. A. Comunicante posterior. 6. Giro parahipocampal.

\section{DISCUSIÓN}

La hipoplasia o agenesia y la incidencia de aneurismas en la ACA (A1) reportados por Karazincir et al. (2004) entre otros, se debería según Fujimoto \& Tanaka (1989) a las variaciones observadas en el círculo arterial del cerebro. (Fig. 5). La presencia de $22 \%$ de ACA hipoplástica reportada por Marinkovic et al. (1986) y de $26,6 \%$ en pacientes con aneurismas por Karazincir et al., son valores muy superiores al 6\% observado en nuestra investigación.

Aydin et al. (1997) informaron que el 60\% de los pacientes operados presenta variaciones anatómicas en la vecindad de la arteria comunicante anterior, dentro de las cuales hay un $8,3 \%$, dobles o triples en pacientes con aneurismas (Fig. 5).

El calibre de la ACI señalado por Saeki \& Rhoton es de 4,3 $\mathrm{mm}$ sin diferenciar lado y sexo. Esta variable fue registrada en nuestro trabajo con 3,84mm en el lado derecho y de 3,96 mm en el lado izquierdo, aproximándose a los valores mencionados.

Al analizar parte de la estructura del círculo arterial del cerebro, sus dimensiones, lados, sexo e índice cefálico, es evidente su variabilidad individual entre las arterias.

MANDIOLA, E.; ALARCÓN, E., OÑATE, J. C., SANHUEZA, P.; DEL SOL, M. \& OLAVE, E. Biometrical characteristics of the anterior cerebral artery in its proximal segment (A1) and internal carotid. Int. J. Morphol., 25(4):915-918, 2007.

SUMMARY: The conformation of the arterial circle of the base of the brain has relevancy in the neurochirurgical clinic as the complex relation that there present the arteries that originate it and its great variability. Due to the high frequency the aneurysms observed in the anterior communicating arteries, besides too, in the precommunicating segment (A1) of the anterior cerebral artery, we have decided to carry out a biometrical analysis of these arteries and the ICA for research possible association or correlation in these arteries. The work was realized in 36 available brains in the anatomical laboratories. These specimens had bioanthropological data. The segment A1 of the anterior cerebral artery observed a length of $12,86 \pm 1.58 \mathrm{~mm}$ and presents a diameter of $2.37 \pm 0.68$ $\mathrm{mm}$ in the right side. In the left side the length of this one segment is $12.62 \pm 1.96 \mathrm{~mm}$ and presents a diameter of $2.42 \pm 0.75 \mathrm{~mm}$. The external diameter of the ACI in the right side was $3.84 \pm 0.68$ $\mathrm{mm}$ and in left side was $3.96 \pm 0.87 \mathrm{~mm}$. It is observed variability of the components that constitute the arterial cerebral circle when it is considered to be the side, the sex and cephalic index.

KEY WORDS; Anterior cerebral artery; Cerebral artery circle; Internal carotide artery. 


\section{REFERENCIAS BIBLIOGRAFICAS}

Aydin, I. H.; Tackei, E.; Kadioglu, H.; Tuzun, Y.; Kayaoglu, C. R. \& Barlas, E. Vascular variations associated with anterior communicating artery aneurysms-an intraoperatory study. Minim. Invasive Neurosurg., 40(1):17-21, 1997.

Barbosa, L.; Purriel, J. A.; Meerhoff, W. \& Medoc, J. El polígono de Willis y sus variaciones. Acta Neurol.Latinoamer., 15:224-36, 1969

Carpenter, M. Neuroanatomia Humana. 7. ed. Rio de Janeiro, Interamericana, 1978. pp. 581-90.

Duroux, M. M.P.E.; Dujol, H.; Avet, J. \& Gabrielle, C. A propos du polygone de Willis. C.R. Ass, Anat., 79: 328-338, 1953

Fujimoto, K. \& Tanaka,O. Morphological examination of the circulus arteriosus cerebri human (circle of Willis). Anterior and posterior communicating arteries. Anat. Anz., 168 (2):145-54, 1989

Godinov, V. M. The arterial System of the Brain. Am. J. Phys. Anthrop., 13 (3):359-84, 1929.

Gonzalez, A. A. Círculo arterial de Willis. Sus medidas externas y variaciones anatómicas. Acta Neurol. Latinoamer., 5:1-6, 1959

Gulisano, M.; Zecchi, S.; Pacini, P. \& Orlandini, O. The Behaviour of some human arteries as regards the conected circunference. A statistical research. Anat. Anz. 152:34157,1982

Hillen, B. \& Hoogstraten, H.W. Functional Anatomy of the circulus arterius cerebri. Bull.Asoc. Anat. 74(227):72, 1990

Kamath, S. Observations en the length and diameter of vessels forming the circle of Willis. J. Anat., 133 (3):419-23, 1981

Karansincir, S.; Ada, E.;Sarilma, Z. A.:Yalcin, O.; Vidinl, I. B. $\&$ Sabin. Frequency of vascular variations and anomalies accompanying intracraneal aneurysms. Tani. Girisim Radyol., 10(2):103-9, 2004.

Kim, Ge.; Cho, Yp. \& Lim, Sm. The anatomy of the circle of Willis as a predictive factor for intra-operative cerebral ischemia (shunt need) during carotid endarterectomy. Neurol Res., 24(3):237-40, 2002.

Krupa, B. \& Krupa, U. Variations of the anterior communicating artery. Folia Morphol., 57(3):233-40, 1998
Lazorthes, G.: Gouaze, A.; Santini, J-J. \& Salamon, G. Le cercle arteriel du cerveau . (Circulus arteriosus cerebri). Anat. Clinica, 1:241-57, 1979.

Macchi, C.; Lova, R. M.; Miniati, B.; Gulisano, M.; Pratesi, C.; Conti, A. \& Gensini, G. F. The circle of Willis in healthy older persons. J. Cardiovasc Surg., 43(6):887-90, 2002.

Mandiola, E.; del Sol, M. \& Prates, J. C. Relación Anatómica de la arteria cerebelar superior con los nervios oculomotor y troclear considerando el grupo étnico e índice cefálico. Rev. Chil. Cs. Med. Biol., 5(1):5-10, 1995.

Mandiola, E.; del Sol, M.; Sanz, M. E.; Olave, E.; Gabrielli, C.; Prates, J. C. Distribución arterial en la fisura horizontal del cerebelo. Rev. Chil. Anat.,14(2):177-81,1996.

Mandiola, E.; del Sol, M.; Olave, E.; Gabrielli, C.; Mizusaki, C. \& Prates, J.C. Bifurcation of the basilar artery and its relationship with the superior cerebellar artery at the proximal portion of the anterior pontine segment. Braz. J. Morphol.Sci., 15(1):29- 33, 1998.

Mandiola, E.; del Sol, M.; Sanz, M. E.; Olave, E.; Gabrielli, C. \& Prates, J. C. Variabilidad anatómica de las ramas medial y lateral de la arteria cerebelar superior. Rev. Chil.Anat.,15 (1):85-91, 1997.

Mandiola, E.; Sanz, M. E.; Reumay, P.; del Sol, M.; Olave, E. \& Prates, J. C. Biometría de la arteria cerebelar anteriorinferior en el segmento pontino-anterior y lateral. Rev. Chil. Anat., 17 (2):161-7, 1999.

Milisavljevic, M.; Marinkovic, S.; Lolic-Draganic, V. \& Djordjevic, L. Anastomoses in the Territory of the Posterior Cerebral Arteries. Acta Anat., 127:122-5, 1986.

Saeki, N. \& Rhoton, A. Microsurgical anatomy of the upper basilar artery and the posterior circle of Willis. $J$. Neurosurg., 46:563-78, 1977.

Dirección para correspondencia:

Prof. Dr. Eduardo Mandiola

Facultad de Ciencias de la Salud

Depto. de Ciencias Biológicas

Universidad Andes Bello,

Republica 217, Piso 2

Santiago - CHILE

E-mail: emandiola@unab.cl

Recibido : 14-06-2007 Aceptado: 26-09-2007 